\title{
Produção de biomassa, teor e composição do óleo essencial de Mentha x piperita L. em resposta a fontes e doses de nitrogênio
}

\author{
DESCHAMPS, C. ${ }^{1 *}$; MONTEIRO, R. ${ }^{1}$; MACHADO, M.P. ${ }^{1}$; BIZZO, H. ${ }^{2}$; BIASI, L.A. ${ }^{1}$ \\ ${ }^{1}$ Universidade Federal do Paraná/Departamento de Fitotecnia e Fitossanitarismo, CEP: 81531990, Curitiba-Brasil \\ ${ }^{2}$ EMBRAPA/Agroindústria de Alimentos, CEP: 23020-470, Rio de Janeiro-Brasil *cicero@ufpr.br
}

\begin{abstract}
RESUMO: A adubação nitrogenada influencia o teor e a qualidade dos óleos essenciais de plantas aromáticas. O objetivo deste trabalho foi avaliar a influência de diferentes fontes e doses de nitrogênio na produção de biomassa e teor do óleo essencial de $M$. x piperita. O delineamento experimental foi de blocos casualizados em esquema fatorial $2 \times 3$ (duas fontes de nitrogênio $e$ três doses). A aplicação nitrogenada de cobertura foi realizada 30 dias após o transplante das mudas. O óleo essencial foi extraído pelo processo de hidrodestilação em aparelho graduado Clevenger e sua composição química foi analisada por cromatografia gasosa acoplada à espectrometria de massa. As fontes e doses de nitrogênio não influenciaram significativamente a biomassa de folhas e ramos, assim como a produtividade e o teor do óleo essencial. No entanto, a fonte de nitrogênio pode alterar significativamente a composição do óleo essencial de $M$. $\mathrm{x}$ piperita. O teor de mentol e neomentol foi menor quando $40 \mathrm{~kg} \mathrm{ha}^{-1}$ de uréia foi aplicada, em comparação com a mesma dose de sulfato de amônio. Os teores de mentona foram influenciados apenas pela fonte de nitrogênio, onde níveis mais elevados foram observados quando o sulfato de amônio foi aplicado. Nenhuma alteração nos teores de mentofurano foi encontrada para as diferentes fontes e doses de nitrogênio. Considerando a grande importância econômica de mentol presente no óleo essencial de menta, os resultados obtidos indicaram que a utilização de $20 \mathrm{~kg} \mathrm{ha}^{-1} \mathrm{de}$ nitrogênio é suficiente para garantir a boa produtividade de óleo essencial e alto teor de mentol, e que altas doses de uréia devem ser evitadas.
\end{abstract}

Palavras-chave: adubação nitrogenada, biomassa, extração de óleo essencial, mentol

ABSTRACT: Biomass production, essential oil yield and composition of Mentha x piperita L. according to nitrogen sources and doses. Nitrogen fertilization affects the essential oil yield and composition of aromatic plants. The objective of this work was to evaluate the effect of nitrogen sources and doses on biomass production and essential oil yield and composition of $M$. x piperita $\mathrm{L}$. The experimental design was in randomized blocks with four replications and the treatments in a $2 \times 3$ factorial design, corresponding to two nitrogen sources and three nitrogen doses. The nitrogen topdressing was applied 30 days after planting. The essential oil was obtained by hydrodestilation using a Clevenger apparatus, and the composition was analyzed by gas chromatography and coupled to a mass spectrometry. The nitrogen sources and doses did not significantly affect the leaf and stem biomass, as well as the essential oil yield and productivity. However, the nitrogen source can significantly change the essential oil composition of $M$. x piperita. The level of menthol and neomenthol was lower when $40 \mathrm{~kg} \mathrm{ha}^{-1}$ of urea was applied compared to the same dose of ammonium sulphate. Menthone levels were affected only by the nitrogen source, where higher levels were observed when ammonium sulphate was applied. No changes on menthofurane levels were found for different nitrogen sources and doses. Considering the great economic importance of menthol from mint essential oils, the obtained results indicated that the use of $20 \mathrm{~kg} \mathrm{ha}^{-1}$ of nitrogen is enough to guarantee satisfactory essential oil yield and great menthol levels and that high doses of urea should be avoided.

Key words: nitrogen, biomass, extraction of essential oil, menthol

Recebido para publicação em 19/08/2009

Aceito para publicação em 30/03/2011

Rev. Bras. PI. Med., Botucatu, v.14, n.1, p.12-17, 2012. 


\section{INTRODUÇÃO}

O gênero Mentha (Lamiaceae) ocupa posição de destaque na economia mundial entre as plantas produtoras de óleos essenciais, sendo as principais espécies $M$. x piperita e $M$. arvensis L., que tem como componente majoritário o mentol, composto químico de grande importância para as indústrias farmacêutica, cosmética e alimentícia. A demanda de mentol atualmente ultrapassa 7000 toneladas por ano, movimentando aproximadamente 300 milhões de dólares anualmente (Patel et al., 2007), sendo o maior produtor os Estados Unidos (Tavish \& Harris, 2002).

O crescimento vegetativo da menta é de grande importância na determinação da produtividade das plantas, considerando que a produção e armazenamento do óleo essencial ocorrem em tricomas glandulares peltados, encontrados na parte aérea (Wildung \& Croteau, 2005). Dentre os nutrientes essenciais para o crescimento das plantas está o nitrogênio, fazendo parte da constituição de várias macromoléculas, tais como proteínas, aminoácidos, enzimas, coenzimas, clorofila, ácidos nucléicos e açúcares estruturais em paredes celulares (Taiz \& Zeiger, 2004). Devido às perdas de nitrogênio do solo por lixiviação serem inevitáveis e a mineralização do nitrogênio ser menor do que a quantidade que as plantas precisam, a adubação se torna uma prática importante (Neetson,1995), sendo as principais fontes inorgânicas de nitrogênio a uréia ( $45 \%$ de N) e o sulfato de amônio (20\% de N) (Wiethölter, 2004).

A adubação nitrogenada influencia o teor e a qualidade dos óleos essenciais (Ram et al., 1995; Sangwan et al., 2001); em muitos casos as aplicações nitrogenadas aumentam o teor de óleo essencial em plantas aromáticas, devido o aumento da biomassa por área, área foliar e taxa fotossintética.

Para espécies de menta, a recomendação de adubação do IAC (Van Raij, 1997) considera que ambas $M$. arvensis e $M$. x pipertita, bem como os quimiotipos, apresentam as mesmas exigências nutricionais para acúmulo de biomassa. Além disto, para a exploração comercial do óleo essencial destas espécies não existe até o momento informações relacionadas à adubação que poderiam resultar em maiores teores e composição adequada do óleo essencial. Portanto, o objetivo desse trabalho foi avaliar a influência de diferentes fontes e doses de nitrogênio no teor e qualidade do óleo essencial de Mentha x piperita.

\section{MATERIAL E MÉTODO}

O experimento foi realizado com mudas de M. x piperita formadas a partir de estacas padronizadas com uma gema e meia folha por nó, enraizadas em bandejas contendo substrato comercial Plantmax ${ }^{\circledR} \mathrm{e}$ areia fina (1:1), durante um período de 30 dias, sob condições de temperatura e umidade controladas em casa de vegetação do Departamento de Fitotecnia e Fitossanitarismo da Universidade Federal do Paraná (UFPR).

Após o enraizamento, as mudas foram transplantadas em novembro de 2005 para o Setor de Plantas Medicinais da Estação Experimental do Canguiri, no Município de Pinhais, região Metropolitana de Curitiba, localizada a $25^{\circ} 25^{\prime}$ latitude Sul e 49 $08^{\prime}$ longitude Oeste, com altitude de 930 metros. O solo é caracterizado como Háplico (EMBRAPA, 1999). O clima da região, segundo a classificação de Köeppen, é do tipo "Cfb", mesotérmico úmido sem estação seca definida, com geadas freqüentes no inverno, com temperatura média do mês mais quente inferior a $22^{\circ} \mathrm{C}$. A precipitação média anual da região varia de 1400 a 1500 mm, sendo os meses de abril e maio os mais secos com média da ordem de 75 a $100 \mathrm{~mm}$. A umidade relativa média anual é de 80 a $85 \%$ e a insolação média é de

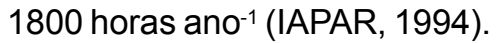

As características químicas do solo no início do experimento foram: P, $218 \mathrm{mg} \mathrm{dm}^{-3}$; M.O., 7,14\%; $\mathrm{K}, 1,16 \mathrm{cmol}_{\mathrm{c}} \mathrm{dm}^{-3} ; \mathrm{Ca}, 11,8 \mathrm{cmol}_{\mathrm{c}} \mathrm{dm}^{-3} ; \mathrm{Mg}, 2,6 \mathrm{cmol}_{\mathrm{c}}$ $\mathrm{dm}^{-3} ; \mathrm{SB}, 15,56 \mathrm{cmol}_{\mathrm{c}} \mathrm{dm}^{-3}$; argila, $45 \% ; \mathrm{V}, 77 \%$ e pH $\left(\mathrm{CaCl}_{2}\right), 5,7$. Valores determinados pelo Laboratório de Análise de Solos (UFPR).

A adubação (NPK) foi realizada uma semana antes do plantio das mudas, e após 30 dias do transplante das mudas foi realizada a adubação nitrogenada de cobertura. A recomendação de adubação foi feita considerando a análise de solo da área experimental, usando como fonte para o cálculo as recomendações do manual do Instituto Agronômico de Campinas (Van Raij, 1997) para o cultivo da menta, variando entre os tratamentos somente a recomendação de nitrogênio, enquanto a de fósforo e potássio foram igualmente aplicadas.

O delineamento experimental foi em blocos ao acaso em esquema fatorial $2 \times 3$ (duas fontes de nitrogênio e três doses), com 16 plantas por parcela de $0,9 \mathrm{~m} \times 1,8 \mathrm{~m}$ e espaçamento entre plantas de $0,3 \mathrm{~m} \times 0,45 \mathrm{~m}$. Os tratamentos consistiram de uréia e sulfato de amônio como fontes de nitrogênio, e doses crescentes a partir da recomendada $(20,30 \mathrm{e}$ $40 \mathrm{kgN} \mathrm{ha}^{-1}$ ).

A colheita foi realizada 120 dias após o plantio, no período da manhã. A área colhida foi de $0,54 \mathrm{~m}^{2} /$ parcela, equivalente à área ocupada por quatro plantas. A parte aérea (ramos e folhas) e estolões (caules adaptados, característico dessa espécie) foram colhidos separadamente. A determinação da biomassa seca foi realizada pela secagem do material vegetal (folhas, ramos e estolões) à temperatura de $65^{\circ} \mathrm{C}$ até peso constante.

Para a extração do óleo essencial foi utilizado o método de hidrodestilação em aparelho graduado

Rev. Bras. PI. Med., Botucatu, v.14, n.1, p.12-17, 2012. 
TABELA 1. Biomassa de folhas e ramos de Mentha x piperita L., em função de duas fontes e três doses de nitrogênio inorgânico.

\begin{tabular}{|c|c|c|c|c|c|c|}
\hline \multirow{3}{*}{ Fontes de $\mathrm{N}$} & \multicolumn{3}{|c|}{ Biomassa de folhas $\left(\mathrm{kg} \mathrm{ha}^{-1}\right)$} & \multicolumn{3}{|c|}{ Biomassa ramos $\left(\mathrm{kg} \mathrm{ha}^{-1}\right)$} \\
\hline & \multicolumn{2}{|c|}{ Doses de $\mathrm{N}\left(\mathrm{kg} \mathrm{ha}^{-1}\right.$} & \multicolumn{2}{|c|}{ Doses de $\mathrm{N}\left(\mathrm{kg} \mathrm{ha}^{-1}\right)$} & \multirow[b]{2}{*}{30} & \multirow[b]{2}{*}{40} \\
\hline & 20 & 30 & 40 & 20 & & \\
\hline Uréia & 1877,6 ns & $1869,5 \mathrm{~ns}$ & $2024,8 \mathrm{~ns}$ & $3727,1 \mathrm{~ns}$ & $3707,0 \mathrm{~ns}$ & $3630,9 \mathrm{~ns}$ \\
\hline Sulfato de amônio & $1677,5 \mathrm{~ns}$ & 1559,9 ns & $2211,1 \mathrm{~ns}$ & $3081,1 \mathrm{~ns}$ & 3446,9 ns & $3365,6 \mathrm{~ns}$ \\
\hline C.V. (\%) & & 23,98 & & & 15,91 & \\
\hline
\end{tabular}

ns - não significativo

de Clevenger, por 120 minutos. O material vegetal, amostras de $100 \mathrm{~g}$ de folhas frescas, foi colocado em balão volumétrico de 2 litros e acrescentado um volume de 1 litro de água destilada. A caracterização química do óleo essencial foi realizada no Laboratório de Análise de Combustíveis Automotivos, do Departamento de Engenharia Química da UFPR, por cromatrografia gasosa acoplada à espectrometria de massa, em cromatógrafo Varian Inc. (modelo CP3800), com detector Saturn 2000 MS/MS e coluna sílica fundida com $30 \mathrm{~m}$ de comprimento (fase estacionária PONA). Utilizou-se hélio como gás de arraste sob pressão da coluna de 30,0 psi. A condição inicial de temperatura era de $60^{\circ} \mathrm{C}$, com elevação da temperatura a $90^{\circ} \mathrm{C}$ na razão de $3^{\circ} \mathrm{C}$ permanecendo por 5 minutos, elevação da temperatura a $140^{\circ} \mathrm{C}$ na razão de $3^{\circ} \mathrm{C}$, elevação da temperatura a $240^{\circ} \mathrm{C}$ na razão de $30^{\circ} \mathrm{C}$ permanecendo por 5 minutos. O volume de $0,1 \mu \mathrm{L}$ de óleo essencial foi injetado com razão de split 200 e temperatura de injeção de $250^{\circ} \mathrm{C}$. A identificação dos constituintes químicos foi realizada comparando-se à biblioteca da Nist 98 (Varian Inc.).

Os dados foram submetidos à Análise de Variância (ANOVA), teste $F(\alpha=5 \%)$ e teste de Tukey para significância das diferenças entre médias. Os dados em porcentagem foram transformados para arc sen $\sqrt{x / 100}$. Utilizou-se o software ASSISTAT.

\section{RESULTADO E DISCUSSÃO}

As fontes e doses de nitrogênio não influenciaram significativamente a produção de biomassa de folhas e ramos. No entanto, a partir dos dados apresentados na Tabela 1, observa-se que 0 aumento das doses de nitrogênio aplicadas na forma de uréia e sulfato de amônio, aumentaram a biomassa das folhas, sendo obtida a maior biomassa de folhas $\left(2211,1 \mathrm{~kg} \mathrm{ha}^{-1}\right)$ com o sulfato de amônio na dose de $40 \mathrm{~kg} \mathrm{ha}^{-1}$ de nitrogênio. Isso mostra que o nitrogênio pode interferir de alguma forma na biomassa foliar de $M$. x piperita, Em espécies como o manjericão, não foi observado aumento na biomassa de folhas quando testado o efeito de nitrogênio inorgânico, nas formas de nitrato e amônio (Adler et al.,1989), e, além disso, a ausência de $\mathrm{N}$ e $\mathrm{P}$ reduziu o crescimento de $M$. x piperita (Praszna \& Bernath, 1993).

A razão folhas:ramos foi em média 0,54 , não tendo influência das diferentes fontes e doses de nitrogênio. Para Mentha arvensis ocorreu redução na razão folhas:ramos com o aumento de nitrogênio, sendo este fato relacionado com o maior crescimento da planta, causando maior senescência nas folhas inferiores devido ao sombreamento (Ram \& Kumar, 1997). Resultados semelhantes ao desse trabalho foram encontrados em manjericão, em que os diferentes níveis de nitrogênio testados não diferiram para a razão folhas:ramos (Sifola \& Barbieri, 2006).

As fontes e doses de nitrogênio não apresentaram efeito significativo para o teor $e$ produtividade de óleo essencial. Porém, observase redução do teor de óleo essencial com o aumento da dose de nitrogênio de 20 para $40 \mathrm{~kg}$ $\mathrm{ha}^{-1}$, nas duas fontes testadas, obtendo-se os maiores teores (aproximadamente $40 \mu \mathrm{L} \mathrm{g}^{-1} \mathrm{~ms}$ ) com as doses de $20 \mathrm{~kg} \mathrm{ha}^{-1}$ de nitrogênio na forma de uréia e $30 \mathrm{~kg} \mathrm{ha}^{-1}$ de nitrogênio na forma de sulfato de amônio (Tabela 2). Kiran \& Patra (2003) observaram maiores produção de biomassa e teor de óleo essencial em $M$. arvensis quando utilizaram diferentes materiais como retardantes de nitrificação, provavelmente devido à inibição da hidrólise da uréia, levando a uma redução na perda de $\mathrm{N}$ do solo e prolongamento do período de utilização do $\mathrm{N}$ aplicado.

A interação positiva para teor de óleo essencial e biomassa, entre adubação orgânica e mineral, foi relatada por Patra et al. (2000), Kiran \& Patra (2003) e Oliveira Junior et al. (2005), em solos com baixos teores de matéria orgânica e argila, condições diferentes do solo do presente experimento, rico em matéria orgânica e argiloso.

O tempo de cultivo também está associado ao teor de óleo essencial, e para M. piperita a colheita pode ser realizada entre 60 e 120 dias (Valmorbida et al., 2006). Maior teor de óleo essencial foi obtido em M. x piperita aos 70 dias

Rev. Bras. PI. Med., Botucatu, v.14, n.1, p.12-17, 2012. 
de cultivo (Marcum e Hanson, 2006), assim como para M. arvensis (Czepak, 1998) e M. x villosa Huds (Inneco et al., 2003) obteve-se os maiores teores de óleo essencial e óxido de piperitona entre 80 e 95 dias de cultivo.

A produtividade de óleo essencial foi reduzida com o aumento da dose de nitrogênio quando utilizada como fonte a uréia, de 77,0 $\mathrm{L} \mathrm{ha}^{-1}$ para 58,9 $\mathrm{L} \mathrm{ha}^{-1}$. Com o sulfato de amônio o aumento da dose de nitrogênio elevou a produtividade de óleo essencial de $65,9 \mathrm{~L} \mathrm{ha}^{-1}$ para $81,8 \mathrm{~L} \mathrm{ha}^{-1}$ (Tabela 2).

Houve interação entre as fontes e doses de nitrogênio para a porcentagem de mentol e neomentol. As doses de nitrogênio influenciaram a porcentagem de mentol, ocorrendo redução na dose mais elevada $\left(40 \mathrm{~kg} \mathrm{ha}^{-1}\right)$ na forma de uréia. Obtendo-se as maiores porcentagem de mentol ao aplicar 20 ou $30 \mathrm{~kg} \mathrm{ha}^{-1}$ de nitrogênio na forma de uréia e sulfato de amônio (Tabela 3). Este valor foi próximo ao obtido por Scavroni et al.(2006) para a mesma espécie. Contudo, em Mentha piperita hidropônica, Souza et al.(2006) obtiveram apenas $5-8 \%$ de mentol, atribuindo este resultado à idade da planta (50 dias de cultivo), devido às folhas jovens conterem mais monoterpenos em alto estado de oxidação e folhas mais velhas apresentarem monoterpenos com baixo estado de oxidação. Da mesma forma Croteau et al. (2000) relatam que o conteúdo de mentol em $M$. piperita aumenta com a maturação das folhas.

O teor de mentol e neomentol foi menor quando $40 \mathrm{~kg} \mathrm{ha}^{-1}$ de uréia foi aplicada, em comparação com a mesma dose de sulfato de amônio (Tabela 3). Na rota da biossíntese de mentol a pulegona pode formar a mentona, que forma 0 neomentol ou o mentol (Croteau et al., 2000).

Não houve efeito significativo da uréia e do sulfato de amônio nas doses testadas para a porcentagem de mentofurano (Tabela 4). Para que um óleo essencial seja considerado de boa qualidade este deve conter mais de $40 \%$ de mentol, 18 a $25 \%$ de mentona e menos de $6 \%$ de mentofurano (I.T.E.I.P.M.A.I., 1989). Burbott \& Loomis (1967), encontraram nas inflorescências de Mentha x piperita, altos teores de mentofurano e pulegona, mas durante o florescimento das plantas, encontraram nas folhas maior teor de mentol e mentona. Nos resultados encontrados, nenhuma alteração nos teores de mentofurano foi encontrada para as diferentes fontes e doses de nitrogênio.

Somente as fontes de nitrogênio tiveram efeito significativo na porcentagem de mentona, sendo a uréia superior ao sulfato de amônio $(21,8 \%$ e 19,2\% respectivamente) (Tabela 4).

TABELA 2. Teor e produtividade de óleo essencial de Mentha x piperita L. em função de duas fontes e três doses de nitrogênio.

\begin{tabular}{ccccccc}
\hline & \multicolumn{3}{c}{ Teor de óleo essencial $\left(\mathrm{mL} \mathrm{g}^{-1} \mathrm{~ms}\right)$} & \multicolumn{4}{c}{ Produtividade de óleo essencial $\left(\mathrm{L} \mathrm{ha}^{-1}\right)$} \\
Fontes de N & \multicolumn{3}{c}{ Doses de N $\left(\mathrm{kg} \mathrm{ha}^{-1}\right)$} & \multicolumn{2}{c}{ Doses de N $\left(\mathrm{kg} \mathrm{ha}^{-1}\right)$} \\
& 20 & 30 & 40 & 20 & 30 & 40 \\
\hline Uréia & $40,4 \mathrm{~ns}$ & $38,0 \mathrm{~ns}$ & $30,5 \mathrm{~ns}$ & $77,0 \mathrm{~ns}$ & $66,2 \mathrm{~ns}$ & $58,9 \mathrm{~ns}$ \\
Sulfato de amônio & $38,4 \mathrm{~ns}$ & $40,5 \mathrm{~ns}$ & $36,7 \mathrm{~ns}$ & $65,9 \mathrm{~ns}$ & $62,9 \mathrm{~ns}$ & $81,8 \mathrm{~ns}$ \\
\hline C.V. (\%) & \multicolumn{3}{c}{30,23} \\
\hline
\end{tabular}

ns - não significativo.

TABELA 3. Porcentagem de mentol e neomentol de $M$. x piperita L., em função de duas fontes e três doses de nitrogênio.

\begin{tabular}{ccccccc}
\hline \multirow{2}{*}{ Fontes de N } & \multicolumn{3}{c}{ Mentol (\%) $)^{1}$} & \multicolumn{3}{c}{ Neomentol $(\%)^{1}$} \\
& \multicolumn{2}{c}{ Doses de N $\left(\mathrm{kg} \mathrm{ha}^{-1}\right)^{2}$} & \multicolumn{2}{c}{ Doses de N $\left(\mathrm{kg} \mathrm{ha}^{-1}\right)^{2}$} \\
& 20 & 30 & 40 & 20 & 30 & 40 \\
\hline Uréia & $42,2 \mathrm{aA}$ & $37,8 \mathrm{aAB}$ & $34,7 \mathrm{bB}$ & $4,0 \mathrm{aA}$ & $3,5 \mathrm{aAB}$ & $3,3 \mathrm{bB}$ \\
Sulfato de amônio & $39,2 \mathrm{aA}$ & $40,9 \mathrm{aA}$ & $39,5 \mathrm{aA}$ & $3,7 \mathrm{aA}$ & $3,7 \mathrm{aA}$ & $3,9 \mathrm{aA}$ \\
\hline C.V. (\%) & \multicolumn{3}{c}{4,37} & & 4,39 & \\
\hline
\end{tabular}

${ }^{1}$ Dados transformados para arc sen $\sqrt{x / 100}$. 'Letras minúsculas na mesma coluna e letras maiúsculas na mesma linha não diferem estatisticamente pelo teste de Tukey a $5 \%$ de probabilidade.

Rev. Bras. PI. Med., Botucatu, v.14, n.1, p.12-17, 2012. 
TABELA 4. Porcentagem de mentofurano e mentona de $M$. x piperita L., em função de duas fontes e três doses de nitrogênio.

\begin{tabular}{|c|c|c|c|c|}
\hline \multirow{3}{*}{ Fontes de $\mathrm{N}$} & \multicolumn{3}{|c|}{ Mentofurano $(\%)^{1}$} & \multirow{3}{*}{ Mentona $(\%)^{1,2}$} \\
\hline & \multicolumn{3}{|c|}{ Doses de $\mathrm{N}\left(\mathrm{kg} \mathrm{ha}{ }^{-1}\right)$} & \\
\hline & 20 & 30 & 40 & \\
\hline Uréia & $10,8 \mathrm{~ns}$ & $11,4 \mathrm{~ns}$ & $10,7 \mathrm{~ns}$ & 21,8 a \\
\hline Sulfato de amônio & 10,2 ns & $9,4 \mathrm{~ns}$ & 13,6 ns & $19,2 b$ \\
\hline C.V. $(\%)$ & & 13,99 & & 5,63 \\
\hline
\end{tabular}

Tukey a $5 \%$ de probabilidade. ns - não significativo.

Os resultados apresentados neste estudo mostraram que o nitrogênio inorgânico aplicado em plantas de M. x piperita, nas formas de uréia e sulfato de amônio, pode alterar significativamente a qualidade e teor do óleo essencial, sendo que $20 \mathrm{~kg} \mathrm{ha}^{-1} \mathrm{de}$ nitrogênio é suficiente para garantir a boa produtividade de óleo essencial e alto teor de mentol, e altas doses de uréia devem ser evitadas.

\section{REFERÊNCIA}

ADLER, P.R.; SIMON, J.E.; WILCOX, G.E. Nitrogen form alters Sweet Basil growth and essential oil content and composition. HortScience, v.24, n.4, p.789-90, 1989. BURBOTT, A.J.; LOOMIS, W.D. Effects of light and temperature on the monoterpenes of peppermint. Plant Physiology, v.42, n.1, p.20-8, 1967.

CROTEAU, R.; KUTCHAN, T.M.; LEWIS, N.G. Natural products (secundary metabolites). In: BUCHA-NAN, B.B.; GRUISSEM, W.; JONES, R.J. Biochemistry \& Molecular Biology of Plants. Rockville: Courier Companies, 2000. p.1250-318.

CZEPAK, M.P. Produção de óleo bruto e mentol cristalizável em oito frequências de colheita de Menta (Mentha arvensis L.). In: MING, L.C. et al. (Eds.). Plantas medicinais, aromáticas e condimentares: avanços na pesquisa agronômica. Botucatu: UNESP. v.2. 1998. p.5380.

EMBRAPA. Empresa Brasileira de Pesquisa Agropecuária. Centro Nacional de Pesquisa de solos. Sistema Brasileiro de Classificação de Solos. Brasília: Embrapa produção de informação; Rio de Jeneiro: Embrapa Solos, 1999. 412p.

IAPAR. Instituto Agronômico do Paraná. Cartas climáticas do Estado do Paraná. Londrina, documento 18, 1994. 49p.

INNECO, R. et al. Espaçamento, época e número de colheitas em hortelã rasteira (Mentha $\mathrm{x}$ villosa Huds). Revista Ciência Agronômica, v.34, n.2, p. 247-51, 2003. I.T.E.I.P.M.A.I. Fiches Techniques Chemille, n.4. p.1-11, 1989.

KIRAN, U.; PATRA, D.D. Influence of natural essential oils and their by-products as nitrification retarders in regulating nitrogen utilization for Japanese mint in sandy loam soils of subtropical central Índia. Agriculture,
Ecosystems and Environment, v.94, n.2, p.237-45, 2003. MARCUM, D.B.; HANSON, B.R. Effect of irrigation and harvest timing on peppermint oil yield in Califórnia. Agricultural Water Management, v.82, n.1-2, p.118-28, 2006.

OLIVEIRA JUNIOR, A.C. et al. Teor e rendimento de óleo essencial no peso fresco de arnica, em função de calagem e adubação. Horticultura Brasileira, v.23, n.3, p.735-9, 2005.

PATRA, D.D.; ANWAR, M.; CHAND, S. Integrated nutrient management and waste recycling for restoring soil fertility and productivity in Japanese mint and mustard sequence in Uttar Pradesh, India. Agriculture, Ecosystems and Environment, v.80, n.3, p.267-75, 2000.

PATEL, T.; ISHIUJI, Y.; YOSIPOVITCH, G. Menthol: A refreshing look at this ancient compound. Journal of the American Academy of Dermatology, v.57, n.5, p.873-8, 2007.

PRASZNA, L.; BERNATH, J. Correlations between the limited level of nutrition and essencial oil production of peppermint. Acta Horticulturae, n.344, p.278-89, 1993. RAM, M.; RAM, D.; SINGH, S. Irrigation and nitrogen requirements of Bergamot mint on a sandy loam soil under sub-tropical conditions. Agricultural Water Management, v.27, n.1, p.45-54, 1995.

RAM, M.; KUMAR, S. Yield improvement in the regenerated and transplanted mint Mentha arvensis by recycling the organic wastes and manures. Bioresource Technology, v.59, n.2, p.141-9, 1997.

SANGWAN, N.S. et al. Regulation of essential oil production in plants. Plant Growth Regulation, v.6, n.34, p.3-21, 2001. SCAVRONI, J. et al. Rendimento e composição química do óleo essencial de Mentha piperita L. submetida a aplicações de giberelina e citocinina. Revista Brasileira de Plantas Medicinais, v.8, n.4, p.40-3, 2006.

SIFOLA, M.I.; BARBIERI, G. Growth, yield and essential oil contento f three cultivars of basil grown under different levels of nitrogen in the field. Scientia Horticulturae, v.108, p.408-13, 2006.

SOUZA, W.P. et al. Avaliação do teor e da composição química do óleo essencial de Mentha piperita (L.) Huds durante o período diurno em cultivo hidropônico. Revista Brasileira de Plantas Medicinais, v.8, n.4, p.108-11, 2006. TAIZ, L.; ZEIGER, E. Fisiologia vegetal. 3. ed. Porto Alegre: Artmed, 2004. 719p.

TAVISH, H.M.; HARRIS, D. Na economic study of essential oil production in the UK: a case study comparing non-

Rev. Bras. PI. Med., Botucatu, v.14, n.1, p.12-17, 2012. 
UK lavander/ lavandin production and peppermint/ spearmint production with UK production tecniques and costs. For the Government Industry, Forum for Non. Food Crops, 2002. 58p.

VALMORBIDA, J. et al. Rendimento e composição química do óleo essencial de Mentha piperita L. cultivada em solução nutritiva com diferentes concentrações de potássio. Revista Brasileira de Plantas Medicinais, v.8, n.4, p.56-61, 2006.
VAN RAIJ, B. et al. Recomendações de adubação e calagem para o Estado de São Paulo. 2.ed. Campinas: Instituto Agronômico/Fundação IAC, 1997. 285p.

WIETHÖLTER, S. Manual de adubação e calagem para os estados do Rio Grande do Sul e Santa Catarina. 10. ed. Porto Alegre: SBCS - NRS, 2004. 394p.

WILDUNG, M.R.; CROTEAU, R.B. Genetic engineering of peppermint for improved essential oil composition and yield. Transgenic Resource, n.14, p.365-72, 2005. 